\title{
Colonic Motility, Autonomic Function, and Gastrointestinal Hormones under Psychological Stress on Irritable Bowel Syndrome
}

\author{
Shin Fukudo and Jinichi Suzuki \\ Department of Psychosomatic Medicine, Tohoku University \\ School of Medicine, Sendai 980
}

\begin{abstract}
Funudo, S. and Suzuri, J. Colonic Motility, Autonomic Function, and Gastrointestinal Hormones under Psychological Stress on Irritable Bowel Syndrome. Tohoku J. exp. Med., 1987, 151 (4), 373-385 — Effects of an artificial mental stress on colonic motility, autonomic nervous system, and gastrointestinal hormones were examined in patients with irritable bowel syndrome (IBS). The subjects were 20 patients with typical IBS and 12 controls. A transducer was inserted to the sigmoid colon from the anus for measuring colonic intraluminal pressure, and mirror drawing test was loaded as psychological stress. At the same time, coefficient of variation of R-R interval on ECG (CV-RR) was measured and the levels of plasma catecholamines, gastrin, glucagon, and motilin were assessed. Colonic motility showed a significant increase in the IBS patients during the stress compared with that in controls $(p<0.01)$. Motilin also increased significantly in the IBS patients after the stress $(p<0.01)$. CV-RR and motilin revealed positive relationship with colonic motility alteration in the IBS patients although no significant change was detected in controls. These phenomena are thought to be due to autonomic nervous dysfunction and/or gastrointestinal hormonal derrangements induced by psychological stress. It is suggested that organ specificity of the alimentary tract for the stress exists in this disease.__ irritable bowel syndrome; psychological stress; colonic motility; autonomic nervous system; gastrointestinal hormones
\end{abstract}

Since Almy and his coworkers demonstrated colonic motility altered under stress in man, many investigators have been interested in this phenomenon (Almy and Tulin 1947; Almy et al. 1949a, b, 1950). Chaudhary and Truelove (1961b) suggested that the patients with IBS have hyper-reactivity of the colon in response to the parasympathomimetic drugs. Snape et al. $(1976,1977)$ provided considerable evidence that the IBS patients had increased 3-cycle per min slow waves in the basal state of the colonic myoelectrical activity. Other studies indicated that IBS patients showed a prolonged postprandial increase in colonic

Received May 16, 1986 ; accepted for publication February 20, 1987.

This paper was presented at 8th World Congress of International Society of Psychosomatic Medicine, Chicago, 1985. 
spike (Sullivan et al. 1978) and in motor activity (Connell et al. 1965), as observed with administrations of magnesium sulfate (Harvey and Read 1973a) and cholecystokinin (Harvey and Read 1973b). The role of gastrointestinal hormones in IBS also attracted attention. Besterman et al. (1981) measured gastrointestinal hormones during fasting and postprandial phase in IBS patients, and they concluded that the levels of pancreatic polypeptide and neurotensin showed slight but significant difference between IBS patients and controls. On the other hand, relationship between psychological stress and onset or exacerbation of symptoms of IBS has been reported by many authors (Bockus et al. 1928; Chaudhary and Truelove 1962). Drossman et al. (1982) mentioned that IBS patients had a tendency to complain frequently that the stress changed their bowel patterns and produced abdominal pain.

Although there are many reports on the pathogenesis of IBS, no quantitative analysis has been carried out on the colonic motility, autonomic function, and gastrointestinal hormones under psychological stress. The present study was conducted to solve this problem and to investigate those correlations.

\section{Materials and Methods}

Subjects

The subjects of the present study comprised 20 patients ( 7 male and 13 female) with IBS and 12 cases ( 5 male and 7 female) of controls. The age of the IBS patients ranged 1860 (mean, 44.4 years old). The diagnosis of IBS was made according to the criteria proposed by Snape et al. (1976) and Kruis et al. (1984) ; 1) diarrhea, constipation, or their alternation, 2) abdominal pain, 3) initiation or exacerbation of above symptoms temporally related to psychological stress, 4) abscence of occult blood, pathogenic bacteria, parasites, and their ova on stool examination and culture, 5) a negative finding for lactose intolerance, 6) normal picture of barium enema, upper gastrointestinal series, and endoscopy. The IBS group consisted of 8 cases of constipation-predominance, 6 cases of diarrheapredominance, and 6 cases of their alternation. The mean age of the controls was 35.3 years old, and they had no history, no symptoms, nor finding of gastrointestinal problems. Both subjects were selected from inpatients at Tohoku University Hospital.

\section{Design of the study}

All subjects underwent colonofiberscopy without air insufflation. A motility pressure transducer (Kulite, Model 31, Ridgefield, NJ, USA) was inserted to the descending colon under direct vision with the colonofiberscope (Olympus, MB-3R, Tokyo). After removal of the colonofiberscope, the transducer was connected to an amplifier (NEC-Sanei, Biophysiograph 180-4, Tokyo) and in turn to a rectilinear recorder (NEC-Sanei, Rectigraph 8S, Tokyo). Respirations were monitored by a pneumograph belt placed around the chest of the subjects and connected to the amplifier. Colonic intraluminal pressure recording was initiated $60 \mathrm{~min}$ after the removal of the colonofiberscope. After resting for 20 min, mirror drawing test (Cook 1933) was loaded for $10 \mathrm{~min}$ as psychological stress with an electrical equipment (Seiwa ME, PSYMO-CF 502, Osaka). The instrument was designed to let the subject trace a star-shaped mark on the plate with an electric pen and a buzzer sounds intermittently when the tracing line becomes out of the line on the star-shaped mark. The second rest was maintained for 20 min after the performance. Blood specimens were drawn from the median cubital vein to measure plasma concentrations of catecholamines, gastrin, 
glucagon, and motilin before, during, and after the stress. Blood pressure, mean R-R interval on $\mathrm{ECG}$, and $\mathrm{CV}-\mathrm{RR}$ were recorded in addition to these parameters.

Quantification of colonic motility, blood pressure, $C V-R R$, catecholamines, gastrin, glucagon, and motilin

Colonic movement was pursued by indicators of mean amplitude of the pressure waves, percentage of motor activity, colonic motiloity index, and number of waves per minute. Colonic motility index is the product of the mean amplitude multiplied by the percentage of motor activity which is calculated by dividing the sum of the duration of the pressure waves with the time of periods.

Systolic and diastolic blood pressure were measured three times at one-min intervals in the middle of every period by using an electric digital sphingomanometer (Omron, HEM179, Tokyo) and mean value was evaluated.

Mean R-R interval and CV-RR was calculated automatically by a computed analyzer (Fukuda, Autonomic-100, Tokyo) which picked up one hundred of R-R interval and divided standard deviation by mean value.

Sodium heparin sulfate was added to the blood specimens for catecholamines as the first reagent, ethylendiaminetetraacetic acid with aprotinine was for glucagon and motilin, and no reagent was for gastrin. All of the specimens were centrifuged of $5 \mathrm{~min}$ at $3000 \mathrm{rpm}$ to separate the plasma. Catecholamines were assayed by trihydroxy indole method (Miura et al. 1977) in which plasma catecholamines were absorbed to almina, filtrated with acetic acid, cold evapolated, crystalized, and detected by rapid liquid chromatograph. Other plasma samples were analyzed with using the radioimmunoassay by the chloramine-T method for gastrin and by the double antibody method for glucagon and motilin. The intra-assay coefficients of variations of plasma catecholamines, gastrin, glucagon, and motilin were 2.4, $3.2,2.8$, and $11.0 \%$, and the lower limits of sensitivity were $10,1.0,10$, and $10 \mathrm{pg} / \mathrm{ml}$, respectively.

\section{Statistical analysis}

All of the response variables were analyzed and compared under the two conditions ; one was before, during and after the stress, and another was between the IBS patients and controls. Statistical analysis was done by using paired $t$-test at correspondent data, Student $t$-test at independent data with equal variance, and Welch $t$-test at independent with inequal variance because all data showed almost normal distribution. Spearman's rank and Pearson's correlation coefficients were calculated among these data.

\section{Results}

\section{The examples of colonic intraluminal pressure}

Fig. 1 presents the patterns of colonic intraluminal pressure of a control subject and that of a typical case with IBS. No significant change was seen in the control. However, in the IBS patients, periodic contractile activity of the colon was shown during the stress loading and it continued for a while after the stress disappeared in contrast with period of quiescence before the stress.

\section{Colonic motility}

The number of pressure waves per minute showed no significant difference between the IBS patients and controls before the stress, but it increased significantly in the IBS patients during the stress $(p<0.01)$. Frequency of the waves in the IBS patients was $2.62 \pm 0.33$ (mean \pm s.E.) per min; this result 
A. Control

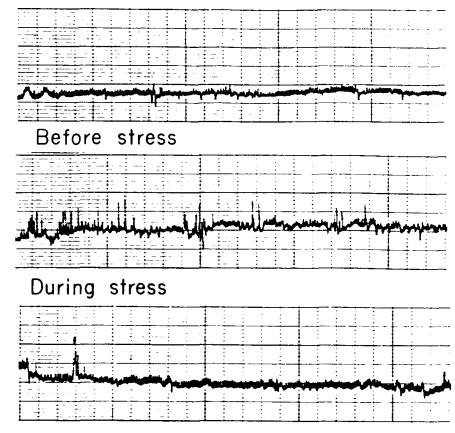

After stress
B. Irritable bowel syndrome

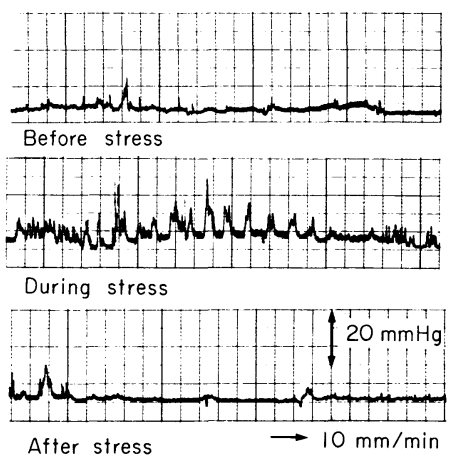

Fig. 1. Real curves of colonic intraluminal pressure in a control subject (A) and a case with irritable bowel syndrome (B) before, during, and after the stress of mirror drawing test.
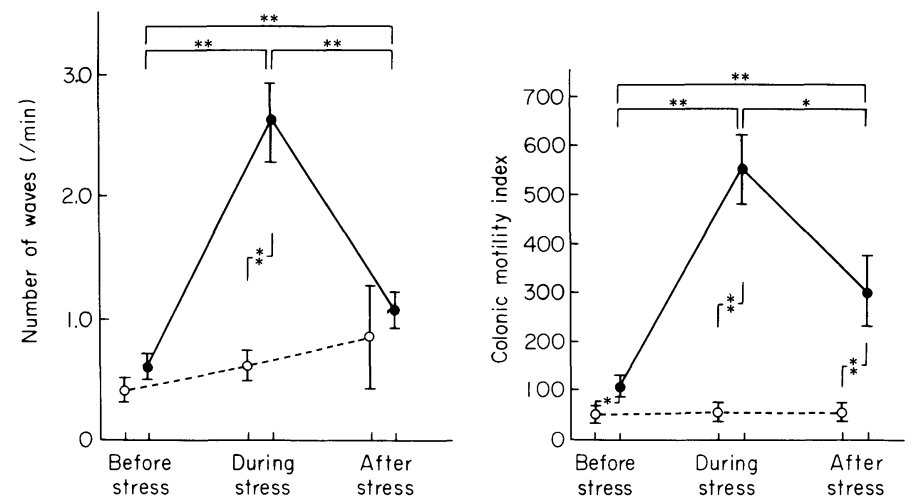

Fig. 2. Variation and difference of colonic motility. Number of pressure waves per min (frequency of waves) and colonic motility index are plotted against the period of the stress.

○... $\quad$ controls $(n=12) ; \bullet-\bullet$ IBS $(n=20) ;{ }^{*} p<0.05 ;{ }^{* *} p<0.01$. Mean \pm s.E.

differed significantly from the findings in controls $(p<0.01$, Fig. $2 a)$.

Mean amplitude of the pressure waves and percentage of motor activity in the IBS patients also increased significantly during the stress $(p<0.01, p<0.01)$. The differences were statistically highly significant as compared with those in controls $(p<0.01)$.

Colonic motility index showed slightly significant difference between the IBS patients and controls before the stress $(p<0.05)$. In the IBS patients, the index increased significantly during the stress $(p<0.01)$ and decreased after the stress $(p<0.05)$, but there was no significant change in controls. These two groups differed significantly during $(p<0.01)$ and after $(p<0.01)$ the stress (Fig. $2 \mathrm{~b})$. 


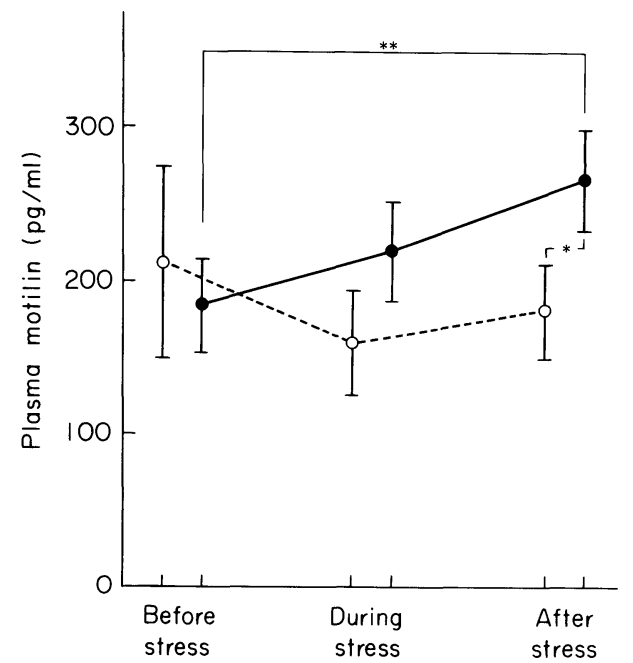

Fig. 3. Variation and difference of plasma motilin concentration. Plasma motilin concentration $(\mathrm{pg} / \mathrm{ml})$ are plotted against the period of the stress. - -... controls $(n=12) ; \bullet-\longrightarrow$, IBS $(n=20) ;{ }^{*} p<0.05 ;{ }^{*} p<0.01$. Mean \pm S.E.

\section{Blood pressure and mean $R-R$ interval}

Systolic and diastolic blood pressure generally tended to increase during the stress and to decrease after the stress, but no significant difference was observed

TABLE 1. Variation and difference of blood pressure (mmHg) and mean $R-R$ interval (msec)

\begin{tabular}{|c|c|c|c|c|c|}
\hline \multirow{2}{*}{ Variables } & \multicolumn{2}{|c|}{ IBS $(n=20)$} & \multicolumn{2}{|c|}{ Controls $(n=12)$} & \multirow{2}{*}{$p$} \\
\hline & Mean & S.E. & Mean & S.E. & \\
\hline \multicolumn{6}{|l|}{ Systolic B.P. } \\
\hline Before & 114.9 & 5.0 & 116.2 & 4.2 & $\mathrm{NS}$ \\
\hline During & 118.5 & 5.6 & $123.8^{*}$ & 4.9 & NS \\
\hline After & $111.5^{*}$ & 4.0 & $115.0^{* *}$ & 4.9 & $\mathrm{NS}$ \\
\hline \multicolumn{6}{|l|}{ Diastolic B.P. } \\
\hline Before & 73.3 & 3.8 & 73.3 & 4.2 & $\mathrm{NS}$ \\
\hline During & $77.7^{* *}$ & 4.4 & $76.6^{*}$ & 3.6 & $\mathrm{NS}$ \\
\hline After & 75.2 & 3.7 & 73.3 & 4.6 & NS \\
\hline \multicolumn{6}{|l|}{ Mean R-R } \\
\hline Before & 742.2 & 29.9 & 643.7 & 41.2 & 0.05 \\
\hline During & $698.6^{* *}$ & 27.3 & 611.6 & 33.0 & 0.05 \\
\hline After & 720.1 & 29.1 & $666.1^{*}$ & 40.0 & $\mathrm{NS}$ \\
\hline
\end{tabular}

${ }^{*} p<0.05$, During-before or during-after difference ; ${ }^{*} p<0.01$, Duringbefore or during-after difference. 
between the IBS patients and controls. Only diastolic blood pressure increased significantly during the stress in the IBS patients $(p<0.01)$. On the other hand, systolic and diastolic blood pressure was elevated significantly in controls $(p<$ $0.05)$.

Mean $\mathrm{R}$ - $\mathrm{R}$ interval during the stress decreased significantly in the IBS patients $(p<0.01)$ and controls $(p<0.05)$. The values in the IBS patients were higher than those in controls before and during the stress $(p<0.05$, Table 1$)$.

$C V-R R$

CV-RR showed no significant change in both groups. Besides, no difference was detected between the IBS patients and controls.

\section{Catecholamines}

Plasma norepinephrine concentration increased significantly in response to the stress in the IBS patients $(p<0.05)$ and also in controls $(p<0.05)$, but plasma epinephrine concentration was affected a little by the stress except the value in the IBS patients after the stress which was more than that before $(p<0.05)$. The increment of catecholamines levels revealed no significant difference between both groups (Table 2).

\section{Gastrin and glucagon}

Plasma concentrations of gastrin and glucagon showed neither significant variation due to the stress nor significant difference between these two groups.

\section{Motilin}

Plasma motilin concentration responded to the stress in the IBS patients. Although the value in controls disclosed no significant change, it increased

TABLE 2. Variation and difference of plasma catecholamines $(\mathrm{pq} / \mathrm{ml})$

\begin{tabular}{llllll}
\hline \multirow{2}{*}{ Variables } & \multicolumn{2}{c}{ IBS $(n=20)$} & \multicolumn{2}{c}{ Controls $(n=12)$} & \multirow{2}{*}{$p$} \\
\cline { 2 - 4 } & \multicolumn{1}{c}{ Mean } & S.E. & Mean & s.E. & \\
\hline Norepinephrine & & & & & \\
$\quad$ Before & 143.3 & 24.7 & 183.2 & 32.6 & NS \\
During & $190.9^{*}$ & 40.3 & 204.7 & 28.6 & NS \\
After & 157.0 & 31.3 & $168.1^{*}$ & 25.2 & NS \\
Epinephrine & & & & & \\
Before & 24.6 & 5.8 & 37.1 & 5.2 & NS \\
During & 27.9 & 5.8 & 31.2 & 4.9 & NS \\
After & $32.9^{* *}$ & 8.8 & 35.9 & 4.9 & NS \\
\hline
\end{tabular}

${ }^{*} p<0.05$, During-before or during-after difference ; ${ }^{* *} p<0.05$, Afterbefore difference. 
significantly after the stress in the IBS patients compared with before the stress $(p<0.01)$. The difference in the increment between the IBS patients and controls showed statistical significance after the stress $(p<0.05$, Fig. 3$)$.

Relationship between each component and colonic motility

Relationship between each component and colonic motility was analyzed statistically as shown in Table 3. Age was not correlated with colonic motility. CV-RR had a tendency to be correlated with colonic motility in the IBS patients during the stress (number of waves: $r=0.45, p<0.05$; colonic motility index : $r=0.44, p<0.05$ ). Pearson's correlation coefficient between CV-RR and colonic motility also revealed significant value (number of waves: $r=0.65, p<0.01$; percentage of motor activity : $r=0.50, p<0.05$ ). However, relationship between CV-RR and colonic motility in controls was not obvious. Norepinephrine and colonic motility tend to correlate inversely in the IBS patients after the stress with statistical significance (number of waves: $r=-0.43, p<0.05$; colonic motility

TABLE 3. Spearman's rank correlation coefficient between each component and colonic motility

\begin{tabular}{|c|c|c|c|c|c|c|}
\hline \multirow{2}{*}{ Variables } & \multicolumn{3}{|c|}{ Number of waves } & \multicolumn{3}{|c|}{ Colonic motility index } \\
\hline & Before & During & After & Before & During & After \\
\hline \multicolumn{7}{|l|}{ Age } \\
\hline IBS & 0.09 & -0.11 & -0.06 & 0.26 & -0.004 & 0.10 \\
\hline Controls & -0.03 & -0.07 & 0.02 & 0.15 & 0.34 & 0.006 \\
\hline \multicolumn{7}{|l|}{ CV-RR } \\
\hline IBS & -0.18 & $0.45^{*}$ & 0.32 & -0.09 & $0.44^{*}$ & -0.79 \\
\hline Controls & 0.25 & -0.13 & 0.05 & -0.28 & -0.55 & 0.28 \\
\hline \multicolumn{7}{|c|}{ Norepinephrine } \\
\hline IBS & 0.14 & -0.16 & $-0.43^{*}$ & -0.04 & -0.20 & $-0.42^{*}$ \\
\hline Controls & 0.31 & -0.07 & -0.02 & 0.48 & 0.44 & -0.05 \\
\hline \multicolumn{7}{|l|}{ Epinephrine } \\
\hline IBS & 0.007 & -0.14 & -0.36 & -0.17 & -0.38 & $-0.48^{*}$ \\
\hline Controls & 0.003 & 0.03 & -0.48 & 0.27 & 0.20 & -0.24 \\
\hline \multicolumn{7}{|l|}{ Gastrin } \\
\hline IBS & 0.11 & -0.07 & -0.44 & -0.21 & 0.04 & -0.46 \\
\hline Controls & 0.40 & -0.20 & -0.20 & 0.001 & -0.08 & 0.20 \\
\hline \multicolumn{7}{|l|}{ Glucagon } \\
\hline IBS & 0.17 & -0.13 & 0.26 & 0.06 & -0.12 & -0.20 \\
\hline Controls & 0.27 & 0.001 & -0.20 & 0.12 & -0.15 & -0.38 \\
\hline \multicolumn{7}{|l|}{ Motilin } \\
\hline IBS & $0.62^{*}$ & -0.26 & 0.40 & $0.44^{*}$ & -0.41 & $0.52^{*}$ \\
\hline Controls & -0.23 & -0.52 & -0.67 & -0.33 & -0.45 & -0.13 \\
\hline
\end{tabular}

${ }^{*} p<0.05 ;$ IBS, $n=20$; Controls, $n=12$. 
index: $r=-0.42, p<0.05)$. Epinephrine and colonic motility index showed moderate but significant negative correlation in the IBS patients after the stress $(r=-0.48, p<0.05)$. There was a lack of a reliable correlation between catecholamines and colonic motility in controls. Neither gastrin nor glucagon was reliably correlated with colonic motility in these two groups. There was no statistical correlation between motilin and colonic motility in controls. However, in the IBS patients, number of waves was directly proportional to motilin significantly before the stress $(r=0.62, p<0.05)$. Colonic motility index also showed a similar correlation of marginal significance with motilin $(r=0.44, p<$ 0.05 , Pearson's $r=0.50, p<0.05)$. These correlations however vanished during the stress. After the stress, colonic motility index was positively correlated with motilin significantly $(r=0.52, p<0.05)$, but number of waves remained a low reliable correlation with motilin. In addition, negative and statistically significant relationship between norepinephrine and CV-RR in the IBS patients before the stress (Pearson's $r=-0.53, p<0.05$ ) was diminished by the stress loading.

Difference in colonic motility and motilin between two groups of the IBS patients with different bowel habits

There was no difference in colonic motility index during the stress between constipation-predominant cases $(673.1 \pm 92.0)$ and diarrhea-predominant cases $(531.8 \pm 139.3)$. No difference was detected in colonic motility index before or after the stress between bowel habits. It was just the same with plasma motilin concentrations.

\section{Judgement of hypermotility of the colon in the IBS patients}

Hypermotility of the colon in response to the stress could be judged when a value of colonic motility index was more than a mean value plus $3 \times$ standard deviation of normal controls. The borderlines were as follows : before the stress, 180 ; during the stress, 220 ; after the stress, 180 . Colonic motility index in 19 cases $(95 \%)$ of the IBS patients showed hypermotility of the colon at least in a phase of the stress but colonic motility index in the rest 1 case $(5 \%)$ did not.

\section{Discussion}

Colonic motility in the IBS patients differed statistically from that in controls during the stress. Mean amplitude, percentage of motor activity, colonic motility index and mumber of waves reflect intensity of contractions, duration of motor activity, degree of colonic movements and frequency of contractile activity. Increases of each parameter during the stress proved the existance of stressinduced hypermotility of the colon in the IBS patients. In controls, colonic motility showed a slight increase during the stress but not significant. Many studies suggest that IBS is associated with abnormal bowel motility in response to 
emotional stress (Kumar and Wingate 1985), meals (Connell et al. 1965), mechanical distension (Whitehead et al. 1980), cholecystokinin (Harvey and Read 1973b), and neostigmine (Chaudhary and Truelove 1961b). Because the onset or exacerbation of symptoms of IBS frequently follows psychological stress (Chaudhary and Truelove 1962), it seems reasonable to assume that abnormal colonic motility is evoked by stress. However, none has proved this phenomenon statistically. Some authors reported that colonic motility showed no significant difference between the IBS patients and controls in response to the stress (Latimer et al. 1981 ; Narducci et al. 1985; Welgan et al. 1985). Discrepancy between these outcomes and ours cannot be explained on the same basis because they have different features; subject selection, method of bowel preparation, the recording site, the recording device, and method of data analysis. A probable reason for this discrepancy could be heterogeneity of IBS. As there was no quantitative diagnostic criteria for functional disturbances of gastrointestinal tract, clinical symptoms and denayal of organic diseases were the only way to make a diagnosis of IBS. Some patients may complain of abdominal symptoms based on the functional disturbances of the gastrointestinal tract, and others may have some symptoms due to psychiatric illness such as hysteria, depression, and anxiety neurosis without pathological basis on the gut (Young et al. 1976; Latimer et al. 1981). Therefore, the examination of colonic motility is useful in discriminating whether a case of IBS really has functional disturbance of the bowel or not. In our study, the trial of quantitative judgement of functional disturbance was not enough to make the new diagnostic criteria for IBS because only one psychological stress (mirror drawing test) was loaded though IBS is considered to have hyperreactivity against various stimuli. There was a great possibility that the 19 cases $(95 \%)$ of the IBS patients had functional disturbance of the bowel and that the remaining 1 case $(5 \%)$ had not. The more cases without functional disturbance are in the experimental subjects of IBS, the less difference in colonic motility between the IBS patients and controls will be observed statistically. Because the IBS patients in our study were considered to consist of mainly psychosomatic features and the psychiatric illness without pathological basis on the gut was tried to be carefully ruled out, the psychological stress would enable to evoke hypermotility of the colon. Besides, some patients with IBS would have spontaneous hypermotility as colonic motility index showed slightly significant differences between the two groups even before the stress. Kumar and Wingate (1985) reported that the IBS patients had total abolition of migrating motor complex under stress and abnormal irregular contractile activity which was spontaneous or evoked by stress in the small bowel. Thus, their report and ours can attribute IBS to a paroxysmal motor disorder which may be spontaneous and/or evoked by stress both in the small bowel and in the colon. Some studies showed the increased frequency of 3 cycle per min colonic contractions in the IBS patients (Snape et al. 1977; Whitehead et al. 1980), and these contractions would be 
nonpropulsive and originate from circular muscle (Snape et al. 1976). Our result of $2.62 \pm 0.33$ cycle per min for frequency of waves in the IBS patients during the stress agreed with their findings.

No difference in colonic motility index and plasma motilin concentration was observed between the IBS patients with different bowel habits. This result is supported by many investigators who have restricted their study groups to patients with abdominal pain (Snape et al. 1976; Whitehead et al. 1980). They have not found that diarrhea-predominant IBS patients have less motility than constipation-predominant patients. Though Connell (1962) stated that constipation was associated with increased activity in the distal colon, whereas diarrhea was associated with decreased contractile activity, Whitehead and Schuster (1985) criticized his report. Their view is held that Connell's diarrhea was pain-less diarrhea whose entity is different from pain-associated diarrhea and which should not be included in the diagnosis of IBS, and that diarrhea-predominant IBS patients have found greater than normal amount of motility in pain-associated diarrhea.

Systolic and diastolic blood pressure elevated during the stress and returned to the original values after the stress and similar observations were made on plasma catecholamines levels and heart rate calculated from mean $R-R$ interval. These results suggest that sympathetic nervous system was excited by the stress in both groups. If excitation of sympathetic nervous system contributes to the pathogenesis of IBS, reactivity or process may differ from control. While high concentration of norepinephrine inhibits colonic motor activity, low concentration of epinephrine stimulates myoelectrical activity (Wienbeck and Christensen 1971) and motor activity of circular muscle of cat colon in vitro (Anuras and Christensen 1981). On the other hand, the difference in mean $R-R$ interval between the IBS patients and controls before and during the stress may originate from the difference in parasympathetic tone. Namely the IBS patients would be more parasympathetic than controls. Indeed, CV-RR, which is thought to be a good indicator of parasympathetic function especially an efferent activity of the cardiac branches of the vagal nerve (Wheeler and Watkins 1973), correlated slightly with colonic motility in the IBS patients during the stress. This finding suggests that parasympathetic excitation affects on hypermotility of the colon in the IBS patients during the stress. It is reasonable and is in agreement with the report by Chaudhary and Truelove (1961a, b). CV-RR, however, failed to show clear variation in response to the stress probably because the parasympathetic fibers in the distal colon are mainly supplied by the pelvic nerves (Davison 1983). Catecholamines showed negative correlation with colonic motility in the IBS patients after the stress. It is considered that excitation of parasympathetic system may calm down gradually with inhibition of colonic motility by accumulation of plasma catecholamines.

Gastrointestinal hormones in IBS have been reported in several articles. 
Besterman et al. (1981) reported that fasting and postprandial levels of gastrin, insulin, gastric inhibitory polypeptide, pancreatic polypeptide, motilin, enteroglucagon, and neurotensin in the IBS patients revealed no overall major abnormality of secretion except pancreatic polypeptide and neurotensin, which differed from controls slightly. Christofides and Bloom (1981) also described no significant difference of fasting and postprandial levels of motilin between the IBS pateints and controls. In our study, however, plasma motilin concentration elevated significantly by the psychological stress in the IBS patients. Because motilin is the only substance known to be active on the gastrointestinal motility during the interdigestive state (Itoh et al. 1976) and because it was shown that an infusion of motilin provoked a significant increase in colonic myoelectric and pressure changes (Rennie et al. 1980), our results suggest that the interdigestive colonic motor activity under the stress may be at least in part influenced by plasma motilin concentration in the IBS patients. Correlation between motilin and colonic motility in the IBS patients was excessively of interest. It varied positive before, no correlation during, and positive after the stress. Because muscular contractile action of motilin was suppressed when cyclic-AMP increased by isobutyltheophylline (phosphodiesterase inhibitor) (Schubert et al. 1975) and catecholamines (adenylate cyclase inducer), muscular contractile action of motilin would be influenced by excitement of sympathetic nervous system. Besides, potentiation of motilin by acetylcholine on smooth muscular contraction of the gut was reported by Strunz et al. (1976). So excitation of the autonomic nervous system may contribute to the alteration of relationship between motilin and colonic motility under the stress in the IBS patients.

In conclusion, it is shown from these data that the psychological stress could induce hypermotility of the colon in the IBS patients via autonomic nervous system and/or gastrointestinal hormones. In autonomic nervous system, parasympathetic excitation would play a chief role, but sympathetic system may also excite and have influence on colonic smooth muscle to some extent. It is also suggested that changing levels of gastrointestinal hormones such as motilin in plasma and altering reactivity of the colonic smooth muscle to them gave rise to this phenomenon.

\section{References}

1) Almy, T.P. \& Tulin, M. (1947) Alterations in colonic function in man under stress : Experimental production of changes simulating the "irritable colon". Gastroenterology, 8, 616-626.

2) Almy, T.P., Kern, F. \& Tulin, M. (1949a) Alterations in colonic function in man under stess. II. Experimental production of sigmoid spasm in healthy pearsons. Gastroenterology, 12, 425-436.

3) Almy, T.P., Hinkle, L.E., Berle, B. \& Kern, F. (1949b) Alterations in colonic function in man under stress. III. Experimental production of sigmoid spasm in patients with spastic constipation. Gastroenterology, 12, 437-449. 
4) Almy, T.P., Abott, F.K. \& Hinkle, L.E. (1950) Alterations in colonic function in man under stress. IV. Hypomotility of the sigmoid colon, and its relationship to the mechanism of functional diarrhea. Gastroenterology, 15, 95-103.

5) Anuras, S. \& Christensen, J. (1981) Effects of autonomic drugs on cat colonic muscle. Amer. J. Physiol., 240, G, 361-364.

6) Besterman, H.S., Sarson, D.L., Rambaud, J.C., Stewart, J.S., Guerin, S. \& Bloom, S.R. (1981) Gut hormone response in the irritable bowel syndrome. Digestion, 21, 219224.

7) Bockus, H.L., Bank, J. \& Willkinson, S.A. (1928) Neurogenic mucous colitis. Amer. J. med. Sci., 176, 813-829.

8) Chaudhary, N.A. \& Truelove, S.C. (1961a) Human colonic motility: A comparative study of normal subjects, patients with ulcerative colitis, and patients with irritable colon syndrome. I. Resting patterns of motility. Gastroenterology, 40, 1-17.

9) Chaudhary, N.A. \& Truelove, S.C. (1961b) Human colonic motility: A comparative study of normal subjects, patients with ulcerative colitis, and patients with irritable colon syndrome. II. The effect of prostigmine. Gastroenterology, 40, 18-26.

10) Chaudhary, N.A. \& Truelove, S.C. (1962) The irritable colon syndrome: A study of clinical features, predisposing causes, and prognosis in 130 cases. Quart. J. Med., 31, 307-323.

11) Christofides, N.D. \& Bloom, S.R. (1981) Motilin. In : Gut hormones, 2nd ed., edited by S.R.Bloom \& J. Polak, Churchill Livingstone, Edinburgh, pp. 273-279.

12) Connell, A.M. (1962) The motility of the pelvic colon. II. Paradoxical motility in diarrhea and constipation. Gut, 3,342-348.

13) Connell, A.M., Jones, F.A. \& Rowlands, A. (1965) Motility of the pelvic colon. IV. Abdominal pain associated with colonic hypermotility after meals. Gut, 6, 105-112.

14) Cook, T.W. (1933) Studies in cross education. I. Mirror tracing of the star-shaped maze. J. exp. Psychol., 16, 144-160.

15) Davison, J.S. (1983) Innervation of the gastrointestinal tract. In: $A$ Guide to Gastrointestinal Motility, edited by J. Christensen \& D.L. Wingate, Wright, Bristol, pp. 1-47.

16) Drossman, D.A., Sandler, R.S., McKee, D.C. \& Lovitz, A.L. (1982) Bowel patterns among subjects not seeking health care. Gastroenterology, 83, 529-534.

17) Harvey, R.F. \& Read, A.E. (1973a) Effect of oral magnesium sulfate on colonic motility and symptoms in patients with the irritable bowel syndrome. Gut, 14, 983987.

18) Harvey, R.F. \& Read, A.E. (1973b) Effect of cholecystokinin on colonic motility and symptoms in patients with the irritable bowel syndrome. Lancet, 1, 1-3.

19) Itoh, Z., Honda, R., Hiwatashi, K., Takeuchi, S., Aizawa, I., Takayanagi, R. \& Couch, E. (1976) Motilin-induced mechanical activity in the canine alimentary tract. Scand. J. Gastroent., 39, 93-110.

20) Kruis, W., Thieme, C.H., Weinzierl, M., Schussler, P., Holl, J. \& Paulus, W. (1984) A diagnostic score for the irritable bowel syndrome. Gastroenterology, 87, 1-7.

21) Kumar, D. \& Wingate, D.L. (1985) The irritable bowel syndrome: A paroxysmal motor disorder. Lancet, 2, 973-977.

22) Latimer, P., Sarna, S., Campbell, D., Latimer, M., Waterfall, W. \& Daniel, E.E. (1981) Colonic motor and myoelectrical activity: A comparative study of normal subjects, psychoneurotic patients, and patients with irritable bowel syndrome. Gastroenterology, 80, 893-901.

23) Miura, Y., Campese, V., DeQuattro, V. \& Meijer, D. (1977) Plasma catecholamines via an improved fluorometric assay: Comparison with an enzymatic method. $J$. Lab. clin. Med., 89, 421-427.

24) Narducci, F., Snape, W.J., Battle, W.M., London, R.L. \& Cohen, S. (1985) Increased colonic motility during exposure to a stressful situation. Dig. Dis. Sci., 30, 40-44. 
25) Rennie, J.A., Christofides, N.D., Mitchenere, P., Johnson, A.G. \& Bloom, S.R. (1980) Motilin and human colonic activity. Gastroenterology, 78, A, 1243.

26) Schubert, E., Mitznegg, P., Strunz, U., Domschke, W., Domschke, S., Wunsch, E., Jaeger, E., Demling, L. \& Heim, F. (1975) Influence of the hormone analogue 13-nle-motilin and 1-methyl-3-isobutylxanthine on tone and cyclic $3{ }^{\prime}, 5^{\prime}$-AMP content of antral and duodenal muscles in the rabbit. Life Sci., 16, 263-272.

27) Snape, W.J., Carlson, G.M. \& Cohen, S. (1976) Colonic myoelectric activity in the irrtable bowel syndrome. Gastroenterology, 70, 326-330.

28) Snape, W.J., Carlson, G.M., Matarazzo, S.A. \& Cohen, S. (1977) Evidence that abnormal myoelectrical activity produces colonic motor dysfunction in the irritable bowel syndrome. Gastroenterology, 72, 383-387.

29) Strunz, U., Domscheke, W., Domscheke, S., Mitznegg, P., Wunsch, E., Jaeger, E. \& Demling, L. (1976) Potentiation between 13-nle-motilin and acetylcholine on rabbit pyloric muscle in vitro. Scand. J. Gastroent., 11, Suppl. 39, 29-33.

30) Sullivan, M.A., Cohen, S. \& Snape, W.J. (1978) Colonic myo electrical activity in irritable bowel syndrome. New Engl. J. Med., 298, 878-883.

31) Welgan, P., Meshkinpour, H. \& Hoeler, F. (1985) The effect of stress on colon motor and electrical activity in irritable bowel sydrome. Psychosom. Med., 47, 139-149.

32) Wheeler, T. \& Watkins, P.J. (1973) Cardiac denervation in diabetes. Brit. med. J., 4, 584-586.

33) Whitehead, W.E. \& Schuster, M.M. (1985) Irritable bowel syndrome: Physiological and psychological mechanisms. In: Gastrointestinal Disorder, Behavioral and Physiological Basis for Treatment, edited by W.E. Whitehead \& M.M. Schuster, Academic Press, New York, pp. 179-209.

34) Whitehead, W.E., Engel, B.T. \& Schuster, M.M. (1980) Irritable bowel syndrome: Physiological and psychological differences between diarrhea-predominant and constipation-predominant patients. Dig. Dis. Sci., 25, 404-413.

35) Wienbeck, M. \& Christensen, J. (1971) Effect of some drugs on electrical activity of the isolated colon of the cat. Gastroenterology, 61, 470-478.

36) Young, S.J., Alpers, D.H., Norland, C.C. \& Wooddruff, R.A. (1976) Psychiatric illness and the irritable bowel syndrome: Practical implications for the primary physician. Gastroenterology, 70, 162-166. 